\title{
Association of the DBH Polymorphism rs3025343 With \\ Smoking Cessation in a Large Population-Based Sample
}

\section{Hirvonen, Katariina}

2017-09

Hirvonen , K, Korhonen , T, Salomaa , V , Mannisto , S \& Kaprio , J 2017 , ' Association of the DBH Polymorphism rs3025343 With Smoking Cessation in a Large Population-Based Sample ' , Nicotine \& Tobacco Research , vol. 19 , no. 9 , pp. 1112-1115 . https://doi.org/10.1093/ntr/ntx066

http://hdl.handle.net/10138/311504

https://doi.org/10.1093/ntr/ntx066

unspecified

acceptedVersion

Downloaded from Helda, University of Helsinki institutional repository.

This is an electronic reprint of the original article.

This reprint may differ from the original in pagination and typographic detail.

Please cite the original version. 
Effect of the DBH polymorphism rs3025343 on smoking cessation in a large

population-based sample

Katariina Hirvonen $(\mathrm{BSC})^{1,3}$, Tellervo Korhonen ${ }^{1,3,4}(\mathrm{PhD})$, Veikko Salomaa ${ }^{2}(\mathrm{MD}, \mathrm{PhD})$, Satu Männistö $(\mathrm{PhD})$, Jaakko Kaprio (MD, PhD) $)^{1,2,3}$

jaakko.kaprio@helsinki.fi

1) Dept. of Public Health, University of Helsinki, Helsinki, Finland

2) Dept. of Health, National Institute for Health and Welfare, Helsinki, Finland.

3) Institute for Molecular Medicine FIMM, University of Helsinki, Helsinki, Finland

4) University of Eastern Finland, Kuopio, Finland 


\section{Abstract}

Introduction

Genetic variations in $D B H$-gene and its surroundings have been shown to associate with smoking behavior including smoking cessation in several studies. In this study we replicate and measure the effect size for association between $D B H$ polymorphism rs3025343 and smoking cessation in a large population-based sample while examining environmental factors that could relate to the association.

Methods

We studied 11,926 adult subjects from four surveys of the National FINRISK Study. The analysis was restricted to either current or former smokers. Logistic and linear regression analyses were conducted to investigate the relationships of the SNP, covariates, smoking cessation and smoking severity (cotinine, CPD). Gene-environment interactions were tested by likelihood-ratio test.

Results

The association between rs3025343 and smoking cessation (prevalence odds ratio, $O R=1.12, p=0.094$, $95 \% \mathrm{Cl}=0.98-1.30$ ) was replicated identically with the GWAS study of The Tobacco and Genetics Consortium $(\mathrm{OR}=1.12,95 \% \mathrm{Cl}=1.08-1.18)$. None of our tested phenotypes significantly influenced the association between rs3025343 and smoking cessation. Overall, marital status, education, depression, alcohol use, selfrated health and COPD showed phenotypic associations with smoking cessation, but the association of various phenotypes with smoking cessation did not vary by genotype.

\section{Conclusions}

The current study replicates the effect size for the association between rs3025343 and smoking cessation despite lack of overall significance due to smaller sample size. We could not show environmental influences on the association of rs3025343 with smoking cessation.

\section{Implications}


Our study replicates the direction of the association of DBH polymorphism rs3025343 with smoking cessation. We could not detect environmental influences on the strength of the association of rs 3025343 with smoking cessation, but the limited power of our analysis needs to be taken into account. 


\section{Introduction}

Quitting smoking is followed by immediate health benefits and a reduced risk of morbidity and mortality. Of the smokers that quit on their own only as few as $4-7 \%$ do not lapse back to smoking within the first year [1]. Even when utilizing cessation treatments, only $15-25 \%$ maintain abstinence for at least one year [2]. A better understanding of the genetic background of smoking cessation may provide the means to develop new and more efficient treatments, especially when the environmental factors are also taken into consideration. Heritable influences have been found for the ability to quit smoking [3-6]. However, the evidence linking the best known single genetic variants to smoking cessation has been conflicting in observation studies and clinical trials [7-9].

Nicotine enhances dopamine release by activating dopaminergic neurons in the mesolimbic reward pathway, which is involved in feeling pleasure when smoking. The $D B H$-gene encodes dopamine $\beta$ hydroxylase, which catalyzes the conversion of dopamine to norepinephrine, and is also associated with smoking and drug dependencies.

The SNP rs3025343, located $23 \mathrm{~kb}$ upstream of $D B H$, is associated with smoking cessation according to a very large GWAS meta-analysis. The study, which included 64,924 ever smokers ( $\mathrm{OR}=1.12,95 \% \mathrm{Cl}=1.08$ 1.18) found the $G$ allele of rs 3025343 to be more common among former than current smokers [10]. Another study with 3,441 chronic obstructive pulmonary disease (COPD) patients of European ancestry replicated this association by demonstrating similar results $(O R=1.24, p=0.015)$ [11]. Both studies were observational.

In a clinical trial reported by Leventhal and colleagues a haplotype of 6 DBH SNPs was found to predict abstinence at the end of cessation treatment and 6-month follow-up in study samples with high nicotine dependence [12]. Another study reported one DBH polymorphism to be associated with smoking severity among male schizophrenic smokers [13]. Although distinct from SNPs found significant in GWA studies, these findings support the hypothesis that $D B H$ is an important candidate gene involved in smoking behavior. 
Our aim was to replicate the result of GWAS regarding rs3025343 and its association with smoking cessation in a large population-based sample. Furthermore, analyses of the relationship between rs3025343 and environmental factors associated with smoking cessation were conducted in order to observe the influence of such environmental factors on the strength of the association of rs 3025343 with smoking cessation. Analyses of per-allele associations were conducted using a random effects method.

\section{Materials and methods}

\section{Study sample}

Our sample was drawn from a Finnish population-based survey, the National FINRISK Study, which was first initiated in 1972 and has since then been carried out every five years using independent samples from four to six different parts of Finland depending on the year of survey [14]. We used data from cohorts 1992, 1997, 2002 and 2007, comprising of 26,582 genotyped persons with known smoking status. Our analysis focused on the 11,926 genotyped subjects ( $41 \%$ women) who were either current smokers $(n=6,578)$ or had quit smoking at least six months prior to the survey $(n=5,348)$. Other self-reported information about their health-related habits, socioeconomic status and health status were obtained (see supplementary material for details on traits and genotyping). The mean age was 47.7 years (SD 12.7, range 25-74).

\section{Statistical analyses}

For testing the associations between covariates and quitting smoking within different genotypes we combined AA and AG genotypes to get two genotype classes; AX \& GG. This was done due to AA genotype being too rare to permit separate analyses as is visualized in Table 1. We conducted logistic and linear regression analyses to investigate the relationships of the SNP, covariates, smoking cessation and smoking severity. To examine whether the associations of the gene with outcome differed significantly by categories of covariates, we fit models with and without an interaction of the SNP and covariate, and tested the 
significance of the change in model fit using a likelihood ratio test to derive the significance of the interaction. Age, gender, region and the year of questionnaire were adjusted in the analysis.

All statistical analyses were performed using Stata 13.1 [15]. We report nominal p-values throughout the manuscript.

\section{Results}

The effect size $(\mathrm{OR}=1.12, \mathrm{p}=0.094,95 \% \mathrm{Cl}=0.98-1.3)$ for association between $\mathrm{rs} 3025343$ and smoking cessation in the FINRISK study sample was replicated identically with the earlier GWA study $(O R=1.12$, $95 \% \mathrm{Cl}=1.08-1.18)$ although the association did not yield statistical significance [10]. This is shown in Table 1 together with genotype information of the study sample. Rs $3025343 \mathrm{G}$ allele was more abundant in Eastern Finland (North-Karelia \& Northern Savo) than elsewhere. Allele frequencies were similar regardless of the survey year.

Table 2 shows the results of the association analysis between smoking cessation and rs3025343 in different phenotypes. While variation in OR-values was observed within phenotype classes, the likelihood-ratio tests show that none of the gene-environment interactions exceeded statistical significance $(p<0.05)$. Thus, it appears that in this study, none of our tested phenotypes affects overall the strength of the association between rs 3025343 and smoking cessation. Within variables, some individual categories showed nominally significant associations: among married persons ( $O R=1.18,95 \% \mathrm{Cl} 1.00$ to 1.39), among those drinking 14 or more drinks weekly $(\mathrm{OR}=1.60,95 \% \mathrm{Cl} 1.01$ to 2.52$)$ and among those living the most eastern province of Finland ( $\mathrm{OR}=1.52,95 \% \mathrm{Cl} 1.05$ to 2.21$)$. After adjustment for multiple testing, none of these is significant. The logistic regression analysis of the rs3025343 genotypes on smoking cessation in different phenotypes examined the strength of the association between phenotype and smoking cessation within the main genotype classes (first two columns of results) and overall (last column) (Supplementary table 1). The associations of phenotypes with smoking cessation were very similar in both genotype groups. 
However, Supplementary table 1 shows certain interesting results independent of genotype. Overall, having a diagnosis of bronchial asthma $(\mathrm{OR}=1.19,95 \% \mathrm{Cl}=0.99-1.43)$ seems to make quitting smoking a little more probable while having COPD $(\mathrm{OR}=0.55,95 \% \mathrm{Cl}=0.46-0.65)$ influences vice versa. Not surprisingly, quitting smoking is more likely for married people than singles, divorced or widowed. The same applies for high educated over low-educated people. Also, the better the self-rated health is, the greater the likelihood of having quit smoking.

Quitting was more likely for people who have never used medication for depression than for those who have (Supplementary table 1). Expectedly, alcohol consumption was also strongly related to smoking cessation; greater the alcohol use, the smaller the likelihood of having quit smoking. The effect is best seen in heavy drinkers $(\mathrm{OR}=0.48)$ while abstainers comprise the reference group.

The rs3025343 allele did not affect cotinine levels $(p=0.78)$ nor CPD significantly $(p=0.21)$ among current smokers, i.e we find no influence of the variant on amount of smoking.

\section{Discussion}

Several studies have reported association between common variants and smoking cessation although most studies have shown only modest associations. Nevertheless, a large meta-analysis has shown significant association between one single nucleotide polymorphism, rs3025343 near DBH gene, and smoking cessation [10]. This finding has been replicated among COPD-patients [11]. Moreover, a candidate gene study reported rs3025343 to be nominally associated with continuous FTND score in European Americans $(p=0.023)$. Based on present knowledge, FTND is one of the strongest predictors of smoking cessation. This indicates that rs3025343 may have an influence on the level of nicotine dependence and further, on smoking cessation [16]. Our study aimed to replicate the effect size for association between rs3025343 and smoking cessation that was reported in the meta-analysis of the Tobacco and Genetics Consortium. We also evaluated the roles and associations of different environmental factors in order to exclude their possible influence on the relationship between the SNP and smoking cessation. The effect size for the association 
between rs3025343 and smoking cessation was identical to that observed in the meta-analysis (OR=1.12, $\mathrm{p}=0.094,95 \% \mathrm{Cl}=0.98-1.3)$ although the association was not statistically significant in our study.

In addition to studies investigating association between rs3025343 and smoking cessation, diverse studies have been carried out about other polymorphisms near or within $D B H$-gene and their relation to smoking cessation, usually through nicotine dependence level [17-21]. Although their results appear somewhat inconsistent, these studies demonstrate the potential role of $D B H$ in smoking-related behaviors. The key limitation of many studies concerning $D B H$ and smoking cessation and main reason for insignificant results is small sample size since even modest genetic influences usually require large study samples to be detected. Many polymorphisms of $D B H$ associated with smoking cessation may be in strong linkage disequilibrium with each other.

Rs3025343 does not cause a change in the amino acid residue in DBH since it is located in an intergenic region. Nevertheless, it could modify gene expression by affecting transcription either directly, through or together with other genetic variants in strong LD. The fact that only one DBH upstream variant (rs1611115) causes $51 \%$ of the variation in plasma-DBH activity in European-Americans strongly supports the conception that intergenic variants have a notable role in the regulation of gene expression [22]. Despite our relatively large total study sample size, minor allele carriers are rather few (7.1\%). Thus, especially when inspecting disease phenotypes, the number of minor allele carriers becomes very small when examining different phenotypes within genotypes. This leads to the statistical power being too low (22\% according to the power analysis) for demonstrating reliable conclusions regarding minor or major allele's specific influences on smoking cessation. This is the main limitation of our study. To increase power to $80 \%$, as many as 36,092 cases and 29,343 controls would be required in total due to the low minor allele frequency. It should also be taken into account that we inspected and ruled out only a few carefully preselected covariates.

It is of interest that the $D B H$ SNP has a different allelic distribution within Finland. The most probable explanation for the deviating allelic distribution is that the gene pool of people in Eastern and Western 
Finland are subject to influence from different regions together with the relatively small and regionally immobile population. Also, the role of that $\mathrm{G}$ allele on smoking cessation appeared to be more significant in North-Karelia $(\mathrm{OR}=1.52,95 \% \mathrm{Cl}=1.05-2.21)$ than elsewhere, which can partly be due to the different allelic distribution. The regional distribution however did not affect our other results, as we adjusted for region in all analyses.

The current study replicates the effect size for the association between rs3025343 and smoking cessation although the association was not statistically significant. In addition we document associations of several covariates with smoking cessation.

\section{Funding}

VS was supported by the Finnish Foundations for Cardiovascular Research. Jaakko Kaprio has been supported by the Academy of Finland (grants 265240, 263278) and by the Sigrid Juselius Foundation.

\section{Declaration of Interests}

Tellervo Korhonen has consulted for Pfizer Finland on nicotine dependence in 2011-2016. Jaakko Kaprio has consulted for Pfizer Finland on nicotine dependence in 2012-2015.

\section{Acknowledgments}

\section{References}

[1] American Cancer Society. Cancer Facts \& Figures 2014. Atlanta, Ga. 2014.

[2] Fiore MC, Jaen CR, Baker TB. Clinical Practice Guideline. 2008. Treating Tobacco Use and Dependence: 2008 Update. 
[3] Xian H, Scherrer JF, Madden PA et al. The heritability of failed smoking cessation and nicotine withdrawal in twins who smoked and attempted to quit. Nicotine Tob Res. 2003;5(2):245-254

[4] Ray R, Mitra N, Baldwin D et al. Convergent evidence that choline acetyltransferase gene variation is associated with prospective smoking cessation and nicotine dependence. Neuropsychopharmacol. 2010;35:1374-1382

[5] Bergen AW, Conti DV, Van Den Berg D et al. Dopamine genes and nicotine dependence in treatmentseeking and community smokers. Neuropsychopharmacol. 2009;34:2252-2264.

[6] Bergen AW, Michel M, Nishita D et al. Drug Metabolizing Enzyme and Transporter Gene Variation, Nicotine Metabolism, Prospective Abstinence, and Cigarette Consumption. Plos One, 2015;7(10)

[7] Chen LS, Baker TB, Jorenby D et al. Genetic variation (CHRNA5), medication (combination nicotine replacement therapy vs. varenicline), and smoking cessation. Drug Alcohol Depen. 2015;154:278-282.

[8] Leung T, Bergen A, Munafò MR, Ruyck KD, Selby P, De Luca V. Effect of the rs1051730-rs16969968 variant and smoking cessation treatment: a meta-analysis. Pharmacogenomics. 2014;16(7):713-720.

[9] Tyndale RF, Zhu AZX, George TP, et al. Lack of Associations of CHRNA5-A3-B4 Genetic Variants with Smoking Cessation Treatment Outcomes in Caucasian Smokers despite Associations with Baseline Smoking. PLoS ONE 10.5, 2015, e0128109. doi:10.1371/journal.pone.0128109.

[10] The Tobacco and Genetics Consortium. Genome-wide meta-analyses identify multiple loci associated with smoking behavior. Nat. Genet. 2010;42(5):441-447. doi:10.1038/ng.571.

[11] Siedlinski M, Cho MH, Bakke P et al. Genome-wide association study of smoking behaviors in COPD patients. Thorax. 2011;66(10):894-902.

[12] Leventhal AM, Lee $W$, Bergen AW et al. Nicotine dependence as a moderator of genetic influences on smoking cessation treatment outcome. Drug Alcohol Depen. 2014;138:109-117 
[13] Zhang XY, Chen DC, Xiu MH et al. Association of functional dopamine-beta-hydroxylase (DBH) 19 bp insertion/deletion polymorphism with smoking severity in male schizophrenic smokers. Schizophr res. $2012 ; 141: 48-53$

[14] Vartiainen E, Laatikainen T, Peltonen $\mathrm{M}$ et al. Thirty-five-year trends in cardiovascular risk factors in Finland. Int J Epidemiol 2010;39:504-518.

[15] StataCorp. Stata Statistical Software: Release 11. College Station, TX: StataCorp LP, 2009

[16] Yang J, Wang S, Yang Z, Hodgkinson CA et al. The contribution of rare and common variants in 30 genes to risk nicotine dependence. Mol Psychiatry. 2014;1-12

[17] McKinney EF, Walton RT, Yudkin P et al. Association between polymorphisms in dopamine metabolic enzymes ant tobacco consumption in smokers. Pharmacogenetics 2000 Aug;10(6):483-491. PubMed PMID: 10975602.

[18] Johnstone EC, Yudkin PL, Hey K et al. Genetic variation in dopaminergic pathways and short-term effectiveness of the nicotine patch. Pharmacogenetics 2004 Feb;14(2):83-90. PubMed PMID: 15077009.

[19] Huang S, Cook DG, Hinks L et al. CYP2A6, MAOA, DBH, DRD4, and 5HT2A genotypes, smoking behaviour and cotinine levels in 1518 UK adolescents. Pharmocogenet Genomics 2005 Dec;15(12):839-850. PubMed PMID: 16272956.

[20] Breitling LP, Twardella D, Hoffmann MM, Witt SH, Treutlein J, Brenner H. Prospective association of dopamine-related polymorphisms with smoking cessation in general care. Pharmacogenomics 2010 Apr;11(4):527-536 doi: 10.2217/pgs.10.1. PubMed PMID: 20350135.

[21] Shiels MS, Huang HY, Hoffman SC et al. A Community-Based Study of Cigarette Smoking Behavior in Relation to Variation in Three Genes Involved in Dopamine Metabolism: Catechol-O-methyltransferase (COMT), Dopamine Beta-Hydroxylase $(D B H)$ and Monoamine Oxidase-A (MAO-A). Prev Med 2008;47(1):116-122. 
[22] Zabetian CP, Anderson GM, Buxbaum SG et al. A Quantitative-Trait Analysis of Human Plasma-

Dopamine B-Hydroxylase Activity: Evidence for a Major Functional Polymorphism at the DBH Locus. Am J Hum Genet 2001;68:515-522 
Table 1. Genotype details of study sample and association between smoking cessation and rs3025343 per allele in Finnish population sample (logistic regression analysis), number of participants in different genotypes, p-value, odds ratio (OR) per allele and $95 \%$ confidence interval $(95 \% \mathrm{CI})$ in columns.

\begin{tabular}{lccccccc} 
& AA & AG & GG & total & $p$ & OR & $95 \% \mathrm{Cl}$ \\
\hline FINRISK & & & & & & & \\
Former & 6 & 351 & 4,991 & 5,348 & & & \\
Current & 9 & 482 & 6,087 & 6,578 & & & \\
All & 15 & 833 & 11,078 & $\mathbf{1 1 , 9 2 6}$ & 0.094 & 1.12 & $0.98,1.29$
\end{tabular}


Table 2. Association between smoking cessation and rs3025343 by strata of different phenotypes in the FINRISK population sample, prevalence odds ratios (ORs) and $95 \%$ confidence intervals (95\% Cls) from logistic regression. Gene-environment interactions; $p$-value for interaction $\left(p_{i}\right)$ from likelihood-ratio test.

\begin{tabular}{|c|c|c|c|c|c|}
\hline Phenotype & OR & $95 \% \mathrm{Cl}$ & $p_{i}$ & $\mathrm{n}$ & $\%$ \\
\hline Sex & & & 0.50 & 11,926 & 100 \\
\hline Male & 1.10 & $0.91,1.33$ & & 7,030 & 59 \\
\hline Female & 1.16 & $0.93,1.45$ & & 4,896 & 41 \\
\hline Marital status & & & 0.62 & 11,909 & 100 \\
\hline Married & 1.18 & $1.00,1.39$ & & 8,749 & 73 \\
\hline Single & 0.89 & $0.59,1.36$ & & 1,533 & 13 \\
\hline Divorced & 1.08 & $0.67,1.75$ & & 1,334 & 11 \\
\hline Widowed & 1.26 & $0.44,3.63$ & & 293 & 2 \\
\hline Education & & & 0.19 & 11,806 & 99 \\
\hline Low & 1.14 & $0.89,1.47$ & & 4,260 & 36 \\
\hline Medium & 1.28 & $0.99,1.64$ & & 4,005 & 34 \\
\hline High & 0.94 & $0.73,1.22$ & & 3,541 & 30 \\
\hline Region & & & & 11,926 & 100 \\
\hline North-Karelia & 1.52 & $1.05,2.21$ & & 2,321 & 19 \\
\hline Northern Savo & 1.15 & $0.80,1.66$ & & 2,313 & 19 \\
\hline Turku-Loimaa & 1.14 & $0.85,1.52$ & & 2,275 & 19 \\
\hline Helsinki-Vantaa & 1.11 & $0.83,1.49$ & & 2,558 & 21 \\
\hline Oulu & 0.91 & $0,61,1.35$ & & 1,648 & 14 \\
\hline Lapland & 0.78 & $0.45,1.37$ & & 811 & 7 \\
\hline Angina pectoris & & & 0.68 & 11,825 & 99 \\
\hline No & 1.12 & $0.97,1.30$ & & 11,295 & 95 \\
\hline Yes & 1.08 & $0.52,2.19$ & & 530 & 4 \\
\hline Bronchial astma & & & 0.40 & 11,814 & 99 \\
\hline No & 1.14 & $0.99,1.33$ & & 11,293 & 95 \\
\hline Yes & 0.80 & $0.42,1.55$ & & 521 & 4 \\
\hline COPD & & & 0.63 & 11,818 & 99 \\
\hline No & 1.14 & $0.98,1.32$ & & 11,157 & 94 \\
\hline Yes & 0.82 & $0.40,1.67$ & & 661 & 6 \\
\hline Self-rated health & & & 0.83 & 11,845 & 99 \\
\hline Very good & 0.98 & $0.65,1.47$ & & 1,330 & 11 \\
\hline Good & 1.19 & $0.95,1.49$ & & 4,932 & 41 \\
\hline Average & 1.18 & $0.93,1.50$ & & 4,402 & 37 \\
\hline Rather poor & 0.97 & $0.58,1.62$ & & 1,083 & 9 \\
\hline Very poor & 0.70 & $0.15,3.27$ & & 98 & 1 \\
\hline Alcohol use during last week & & & & 11,926 & 100 \\
\hline Yes & 1.14 & $0.96,1.35$ & & 8,441 & 71 \\
\hline No & 1.11 & $0.87,1.44$ & & 3,485 & 29 \\
\hline Alcohol use (drinks/week) & & & 0.75 & 11,660 & 98 \\
\hline abstainers & 1.11 & $0.86,1.44$ & & 3,485 & 29 \\
\hline$>0-3$ & 1.10 & $0.78,1.53$ & & 2,024 & 17 \\
\hline$>3-7$ & 1.10 & $0.78,1.54$ & & 2,289 & 19 \\
\hline$>7-14$ & 1.15 & $0.81,1.64$ & & 2,171 & 18 \\
\hline$>14$ & 1.60 & $1.01,2.52$ & & 1,691 & 14 \\
\hline
\end{tabular}


